\title{
Effects of androgen-deprivation therapy on hypercoagulability in prostate cancer patients: A prospective, longitudinal study
}

\author{
Harmanpreet Kaur, PhD; ${ }^{\prime}$ D. Robert Siemens, MD; Angela Black'; Sylvia Robb'; Spencer Barr'; \\ Charles H. Graham, PhD;1 Maha Othman, MD, PhD ${ }^{1,3}$
}

'Department of Biomedical and Molecular Sciences, Queen's University; ' 2Department of Urology, Kingston General Hospital; ${ }^{3}$ School of Baccalaureate Nursing, St Lawrence College; Kingston, ON, Canada

Cite as: Can Urol Assoc J 2017;11 (1-2):33-8. http://dx.doi.org/10.5489/cuaj.3936

See related commentary on page 39 .

\section{Abstract}

Introduction: Androgen-deprivation therapy (ADT) is the mainstay of systemic therapy for advanced prostate cancer (PCa), but has significant adverse effects, including increasing concern for cardiovascular (CV) and thromboembolic (TE) complications. This study carefully investigates any relationship between ADT use and hypercoagulability as a possible mechanism of these adverse effects.

Methods: We performed a prospective, longitudinal study in a cohort of patients with advanced PCa initiating ADT $(n=18)$. Controls included men with biochemical failure after local therapy on watchful waiting $(n=10)$, as well as healthy controls $(n=8)$. Global hemostasis was evaluated using the sensitive global hemostasis assay, thromboelastography (TEG). Patients were evaluated at baseline and every three months for a minimum of 12 months. Results: The results of the TEG studies demonstrated 14/18 (78\%) of advanced PCa patients had evidence of a hypercoagulable state before initiating therapy. Significant baseline hypercoagulability was documented in this cohort compared to the two control groups. ADT did not appear to exacerbate hypercoagulability over time as a whole: only 10/18 (56\%) patients had TEG findings consistent with hypercoagulability at the end of study. However, 3/18 (17\%) PCa patients initiating ADT had significantly new hypercoagulable TEG changes on treatment compared to baseline.

Conclusions: This prospective pilot study demonstrates a complex interaction between ADT and hypercoagulable state in men with advanced PCa. TEG abnormalities were mostly associated with volume of cancer as compared to ADT use; however, it is possible that ADT may lead to hypercoagulability in a subset of men, suggesting that sensitive monitoring of coagulation of men on ADT could help identify those at risk of developing CV/TE complications. Study limitations include the relatively small cohort of men followed after initiating ADT and these results require confirmation in a larger trial to rule out subtle effects on hypercoagulability.

\section{Introduction}

Androgen-deprivation therapy (ADT) is the cornerstone of systemic therapy for men with advanced prostate cancer (PCa) and is achieved either surgically or chemically using either gonadotropin-releasing hormone $(\mathrm{GnRH})$ agonists or antagonists. ${ }^{1}$ Furthermore, antiandrogens that competitively inhibit the binding of testosterone to the androgen receptor (AR), as well as newer generation AR-axis-targeted therapies, are increasingly used in PCa management. ${ }^{2}$ Given the prolonged treatment history of men with advanced $\mathrm{PCa}$, there has been renewed focus on the myriad systemic and metabolic side effects of nearly complete eradication of androgen function on target organs, specifically those of bone health, dyslipidemia, obesity, and insulin resistance, ${ }^{3-5}$ as well as effects on cognitive and psychological functioning. ${ }^{6-8}$

Furthermore, there is mounting evidence of a deleterious role of ADT on cardiovascular (CV) health. ${ }^{9-13}$ Although the literature investigating this association is still controversial secondary to differences in study design, outcomes, and at-risk populations studied, several meta-analyses of observational studies have suggested a link between ADT and CV disease and mortality. ${ }^{14,15}$ This increased risk of CV events has been observed largely during the first year after initiation of testosterone therapy. ${ }^{11-13}$ There have been several proposed mechanisms explaining these more acute physiological changes as compared to the development of metabolic syndrome with prolonged ADT use. ${ }^{14}$

One of these proposed mechanisms includes the induction of a hyercoagulable state with ADT, the basis of which was theorized after the observation that lower testosterone levels in men lead to reduced fibrinolytic activity. ${ }^{16,17}$ Furthermore, it is well-recognized that cancer patients in general are at significantly higher risk of developing thromboembolic events (TE) than healthy individuals. ${ }^{18}$ Several studies have also suggested significant perturbations in hemostasis and thrombotic risks in PCa patients. ${ }^{19-21}$ More recently, our group has shown in a small cross-sectional series that PCa patients on ADT exhibit increased hypercoagulability, with a subsequent association with venous/arterial TE events. ${ }^{22}$ The aim of the current study was to more closely examine a cohort of advanced 
Kaur et al.

PCa patients in a longitudinal study to better determine the contribution of ADT to coagulation status over time.

\section{Methods}

This was a prospective, longitudinal, pilot study of men with advanced PCa initiating ADT due to a diagnosis of metastatic disease or progression of their non-metastatic cancer. An age-matched control group of men with biochemical failure after local therapy who were managed on watchful waiting were also enrolled into the study. A cohort of healthy males with a recent negative prostatic biopsy were used as a control of coagulation status at baseline. All men enrolled had a non-significant recent past history of either CV or TE events.

The study received ethics approval in accordance with Queens University Ethics Review Board and consent forms from each patient were obtained prior to the study. Baseline clinical and laboratory data was obtained prior to initiating ADT, including clinical disease status and basic coagulation tests (such as prothrombin time [PT] and activated partial thromboplastin time [APTT] and platelet counts). Patients were assessed clinically every three months.

Global hemostasis was evaluated at baseline and early (3-6 months), as well as late (12-15 months), after ADT initiation using the sensitive global hemostasis assay, thromboelastography (TEG). TEG was performed using a TEG ${ }^{\circledR} 5000$ Hemostasis System and TEG ${ }^{\circledR}$ Hemostasis Analyzer software version 4.2 (Haemoscope Corporation, Skokie, IL, U.S.) and was performed according to the manufacturer's instructions. Citrated whole blood obtained from the patients $(340 \mu \mathrm{L})$ was recalcified with $20 \mu \mathrm{L}$ of $0.2 \mathrm{M} \mathrm{CaCl}_{2}$ and placed into the TEG cup. Data were collected for 75-90 minutes and the following major parameters were evaluated: $\mathrm{R}$ time, alpha $(\alpha)$ angle, maximum amplitude (MA) and clotting index $(\mathrm{Cl})$, time to clot formation (R), speed of clot propagation $(\alpha)$, rate of clot formation $(K)$, strength/stability of clot $(M A)$, and clotting index $(\mathrm{Cl}$; a value that is based on the four parameters above). As previously described ${ }^{22}$ hypercoagulability in any individual patients was defined as two or more TEG parameters with values beyond one standard deviation of the control. To assess the global difference in coagulability among the different groups, the averages of each TEG parameter among these groups were compared.

\section{Statistical analysis}

Analyses were performed using GraphPad Prism Software 6 (GraphPad Software). The comparative analysis of TEG parameters between controls and baseline of PCa patients was performed using Student's t test, as well as one-way ANOVA with Tukey's post-hoc test. A $p$ values less than 0.05 was considered significant.

\section{Results}

Eighteen patients with advanced PCa initiating ADT were enrolled into the study with a mean age of 75.5 years (range 57-84) and were followed for a minimum of 12 months. Mean prostate-specific antigen (PSA) at initiation of ADT in the cohort was $33.6 \mathrm{ng} / \mathrm{ml}$ (range 0.6-177.9). Five of 18 patients (28\%) had imaging evidence of metastatic disease. Eight patients were prescribed the $\mathrm{GnRH}$ antagonist degarlix, eight received a GnRH agonist, and two patients were exposed to a combination of agonist and antagonist. Testosterone was maintained at castrate levels throughout the trial. Ten patients with a mean age 73.5 years (range 69-80) on watchful waiting after biochemical failure were enrolled as non-ADT controls. Mean PSA of this control group on watchful waiting was $2.64 \mathrm{ng} / \mathrm{ml}$ (range 0.4-7.4).

Compared to eight age-matched healthy men, TEG data demonstrated that 14 of 18 men $(78 \%)$ of men with advanced PCa were already hypercoagulable at baseline, even before initiating ADT. The analysis of TEG parameters using oneway ANOVA showed significant differences between the three cohorts (advanced cancer-ADT, watchful waiting, and healthy controls) in three TEG parameters; alpha angle $(p=0.01), M A(p=0.04)$ and $\mathrm{Cl}(p=0.02)$, but not in $\mathrm{R}$ time $(p=0.06)$. Post-hoc tests showed a significant increase in MA and $\mathrm{Cl}$ in the advanced cancer-ADT group as compared to the healthy controls. The alpha angle parameter was significantly different in the advanced cancer-ADT group vs. the watchful waiting group (Fig. 1).

During the course of ADT treatment, there were no statistically significant differences in alpha angle $(p=0.65), M A$ $(p=0.795)$, and $\mathrm{Cl}(\mathrm{p}=0.348)$ before and after ADT treatment in the advanced cancer-ADT group (Fig. 2). Interestingly, there was a significant increase in R time (reduced hypercoagulability) after 12-15 months of ADT ( $\mathrm{p}=0.0004)$.

Individual patient analysis showed that $14 / 18$ (78\%) of the advanced PCa patients were hypercoagulable prior to ADT. After 3-6 months of treatment, only $11 / 18(61 \%)$ of these patients were hypercoagulable and after 12-15 months of ADT treatment, only 10/18 (56\%) patients demonstrated hypercoagulable TEG tracings. The cohort of men on watchful waiting demonstrated no significant changes in any of the TEG parameters, $R$ time $(p=0.371)$, angle $(p=0.784)$, MA $(p=0.649)$, and $\mathrm{Cl}(p=0.187)$, over the 12-15 months of followup period (Fig. 3). It is to be noted that we did not identify any significant changes in PT $(p=0.06)$, APTT $(p=0.14)$, platelet count $(p=0.35)$, or white blood cell count $(p=0.96)$ over the study period. These data suggest that ADT may not increase hypercogulability over time, at least within the first year of treatment.

Although as a whole cohort there was no significant changes in TEG parameters and defined hypercoagulability associated with ADT use, on an individual basis, there were 


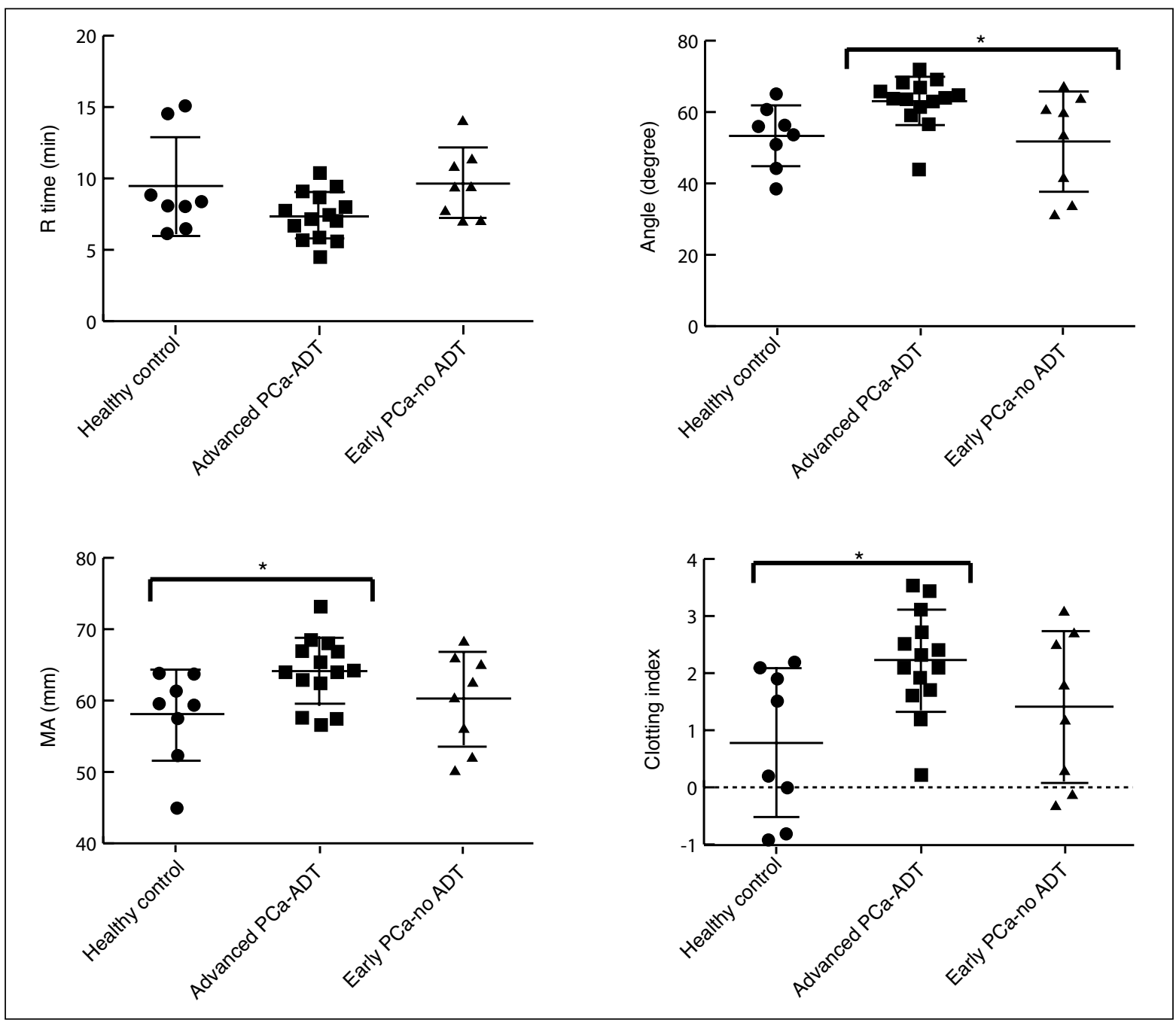

Fig. 1. Comparison of thromboelastography parameters in the different tested groups: advanced prostate cancer (PCa)-androgendeprivation therapy (ADT) before start of treatment; early PCa, no ADT; and healthy, no-cancer control. One-way ANOVA shows a significant increase in maximum amplitude and clotting index in advanced PCa patients before starting ADT as compared to the healthy controls, indicating hypercoagulability and a significant increase in angle as compared to the early cancer group.

3/18 (17\%) PCa patients with normal TEG parameters at baseline who subsequently became hypercoagulable. Two of the three patients had TEG tracing changes on their first assessment within 3-6 months and remained hypercoagulable on the 12-15-month assessment. The third patient was found to be hypercoagulable on the 12-15-month assessment.

We found no significant correlation between PSA levels (as a marker of disease extent and subsequent treatment effect) and degree of coagulability in these patients (baseline: $r=0.0042, p=0.989 ; 3-6$ months: $r=0.055, p=0.841$; 12-15 months: $r=-0.204, p=0.466)$. In some patients with very high serum PSA levels and large volume of disease, no evidence of hypercoagulability was detected, suggesting a complex relationship between ADT, extent of disease, and coagulability in PCa patients.

\section{Discussion}

This prospective, longitudinal, pilot study was designed to systematically examine the relationship between ADT and coagulability in PCa patients with the objective to determine whether hypercoagulability is a measurable adverse effect of ADT, thus contributing to its observed TE/CV risks. Our results demonstrate that this cohort of men with advanced PCa were hypercoagulable before starting ADT as compared to age-matched controls. Over 12-15 months of followup after initiation of ADT, hypercoagulability was not exacerbated in the majority of these patients, with only three patients (17\%) developing a hypercoagulable state after 12 months of treatment.

Metabolic syndrome, including obesity, elevated triglycerides, and reduced high-density lipoprotein (HDL), are established side effects of ADT. ${ }^{23,24}$ All of these well-described 
Kaur et al.



Fig. 2. Thromboelastography parameters before and after androgen-deprivation therapy (ADT) treatment in advanced prostate cancer (PCa)-ADT group over time. One-way ANOVA showed no significant difference in angle, maximum amplitude (MA), or overall clotting index over 12-15 months of treatment (indicating ADT is not associated with hypercoagulability) with a significant increase in R time at 12-15 months after ADT treatment (indicating reduced hypercoagulability).

physiological changes in men on ADT would conceivably lead to higher risk of eventual CV/TE events and mortality. ${ }^{25}$ Hypercoagulability is a direct pathophysiological factor contributing to symptomatic TE and CV complications. ${ }^{26,27}$ However, increased intravascular hypercoagulability, a proposed mechanism of early TE events in cancer patients, has not been examined systematically in association with ADT.

$\mathrm{CV}$ and TE events are common during PCa management, particularly for men with advanced disease requiring systemic therapy.$^{28}$ Previous studies have documented a potential association between ADT and increased venous and arterial TE risk in PCa patients. ${ }^{20,22,29}$ These studies, however, failed to explore the direct impact of ADT on coagulation and the independent role of disease burden. In this current study, we provide evidence that advanced cancer alone may contribute to increased coagulability, as the majority of our cohort demonstrated hyercoagulable TEG findings before initiation of ADT and this did not appear to increase over time. The cohort of PCa patients on watchful waiting did not demonstrate any significant difference in hypercoagulability over 12-15 months' followup. Based on our observations, it is possible that the reduced tumour burden as a result of ADT use in some patients may have improved cancer-induced hypercoagulability over time.
Our data may appear to contradict those of Ziaran et al, who studied 97 patients with locally advanced PCa and showed that after 12 months of ADT, patients had significantly higher fibrinogen, indicating hypercoagulability over time and suggesting increased CV risk in men on ADT. ${ }^{27}$ However, this study did not use TEG as a global, sensitive measure of hypercoagulability and did not include PCa patients on watchful waiting as controls. The findings in the current study may complicate consideration of management of the potential hypercoagulable state in PCa patients relative to previous studies. However, a comprehensive guidance document was recently published and provides specific guidelines on the prevention and treatment of cancer-associated thromboembolism. ${ }^{30}$ Based on this prospective, longitudinal study, hypercoagulability may not be exacerbated by ADT therapy over time, at least not in a majority of patients. Identifying patients at risk, perhaps those with previous history of CV/TE events, may be appropriate to allow more careful monitoring of their coagulation status, and determine those who may benefit from adjuvant anticoagulant prophylaxis. Although one of the strengths of this pilot study is the careful, longitudinal review of coagulation status in men initiating ADT, the cohort size limits the generalizability 

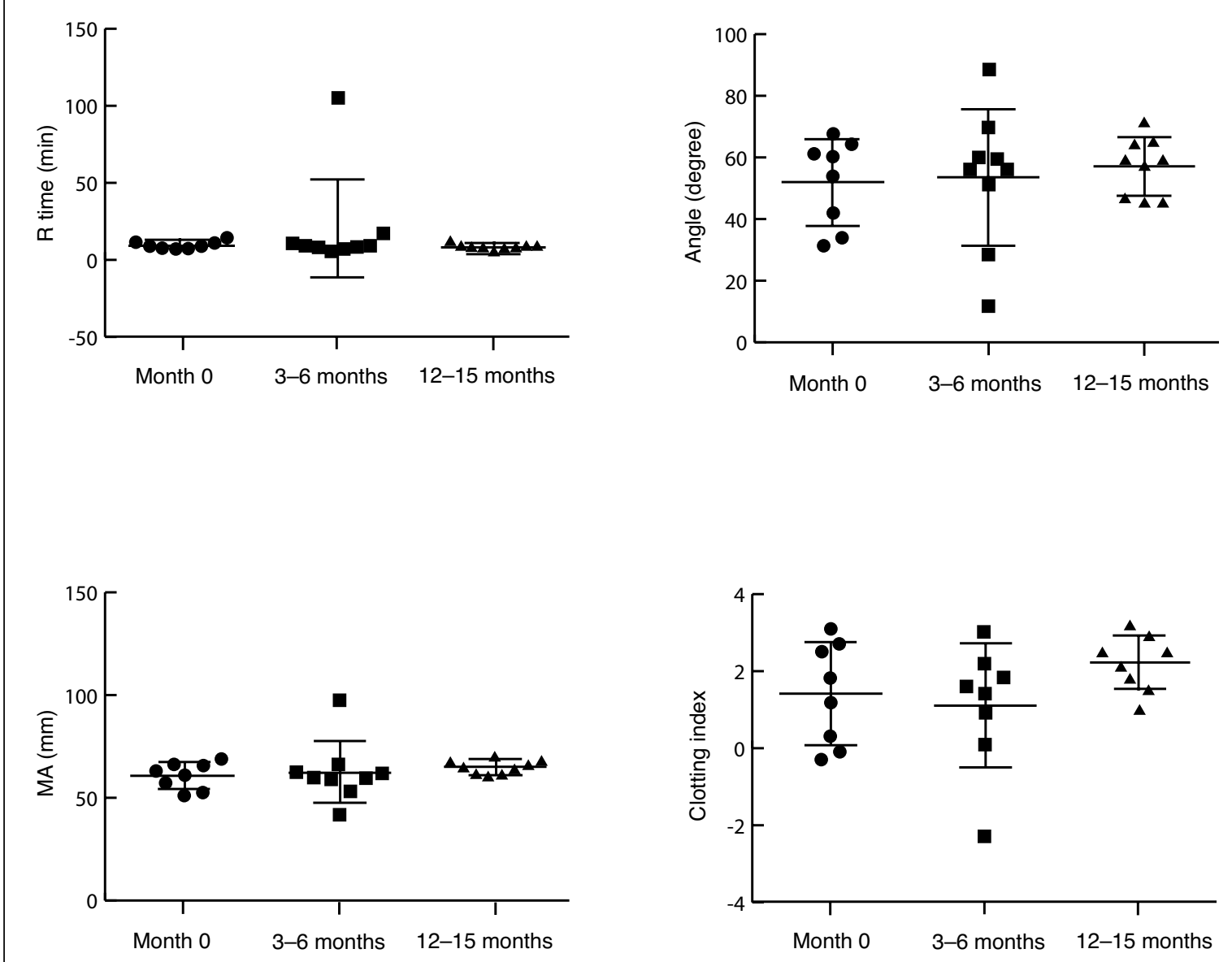

Fig. 3. Thromboelastography (TEG) parameters in the early cancer control group (no androgen-deprivation therapy) over 12-15 months. No significant difference was found in any of the TEG parameters over 12-15 months of treatment. MA: maximum amplitude.

of results for men with different TE risk factors. A larger, prospective evaluation with longer followup would allow a better understanding of more subtle effects of ADT on hypercoagulability and may facilitate an ability to predict those who may suffer TE events.

Competing interests: Dr. Siemens has participated in clinical trials for AbbVie, Astellas, BNIT, Janssen, and Sanofi. The remaining authors report no competing personal or financial interests.

Acknowledgments: We thank the Canadian Hemophilia Society for funding the hemostatic analysis using TEG and Prostate Cancer Canada for funding the clinical component of the study.

This paper has been peer-reviewed.

\section{References}

1. Shore ND, Abrahamsson PA, Anderson J, et al. New considerations for ADT in advanced prostate cancer and the emerging role of GnRH antagonists. Prostate Cancer Prostatic Dis 2013;16:7-15. htrps://doi.org/10.1038/pcan.2012.25
2. Shore ND, Chowdhury S, Villers A, et al. Efficacy and safety of enzalutamide vs. bicalutamide for patients with metastatic prostate cancer (TERRAIN): A randomized, double-blind, phase 2 study. Lancet Oncol 2016;17:153-63. hrtps://doi.org/10.1016/S1470-2045(15)00518-5

3. Saylor PJ, Smith MR. Metabolic complications of androgen-deprivation therapy for prostate cancer. J Urol 2009;181:1998-2006. https://doi.org/10.1016/i.juro.2009.01.047

4. Higano CS. Side effects of androgen deprivation therapy: Monitoring and minimizing toxicity. Urology 2003;61:32-8. https://doi.org/10.1016/S0090-4295(02)02397-X

5. Hakimian P, Blute M Jr, Kashanian J, et al. Metabolic and cardiovascular effects of androgen-deprivation therapy. BJU Int 2008;102:1509-14. https://doi.org/10.1111/i.1464-410X.2008.07933.x

6. Green HJ, Pakenham KI, Headley BC, et al. Altered cognitive function in men treated for prostate cancer with luteinizing hormone-releasing hormone analogues and cyproterone acetate: A randomized, controlled trial. BJU Int 2002;90:427-32. https://doi.org/10.1046/j.1464-410X.2002.02917.x

7. Alibhai SM, Breunis $\mathrm{H}$, Timilshina $\mathrm{N}$, et al. Impact of androgen-deprivation therapy on cognitive function in men with non-metastatic prostate cancer. J Clin Oncol 2010;28:5030-7. htrps://doi.org/10.1200/ $J C 0.2010 .30 .8742$

8. Joly F, Alibhai SM, Galica J, et al. Impact of androgen-deprivation therapy on physical and cognitive function, as well as quality of life of patients with non-metastatic prostate cancer. J Urol 2006; 176:2443-7. htrps://doi.org/10.1016/i.juro.2006.07.151

9. Keating NL, O'Malley AJ, Freedland SJ, et al. Does comorbidity influence the risk of myocardial infarction or diabetes during androgen-deprivation therapy for prostate cancer? Eur Urol 2013;64:159-66. https://doi.org/10.1016/i.eururo.2012.04.035

10. Jespersen $C G$, Norgaard $M$, Borre M. Androgen-deprivation therapy in treatment of prostate cancer and risk of myocardial infarction and stroke: A nationwide Danish population-based cohort study. Eur Urol 2014;65:704-9. https://doi.org/10.1016/i.eururo.2013.02.002 
Kaur et al.

11. D'Amico AV, Denham JW, Crook J, et al. Influence of androgen suppression therapy for prostate cancer on the frequency and timing of fatal myocardial infarctions. J Clin Oncol 2007;25:2420-5. https://doi.org/10.1200/JC0.2006.09.3369

12. Kintzel PE, Chase SL, Schultz LM, et al. Increased risk of metabolic syndrome, diabetes mellitus, and cardiovascular disease in men receiving androgen-deprivation therapy for prostate cancer. Pharmacotherapy 2008;28:1511-22. https://doi.org/10.1592/phco.28.12.1511

13. Ziehr DR, Chen MH, Zhang D, et al. Association of androgen-deprivation therapy with excess cardiac-specific mortality in men with prostate cancer. BJU Int 2015;116:358-65. https://doi.org/10.1111/bju.12905

14. Zhao J, Zhu S, Sun L, et al. Androgen-deprivation therapy for prostate cancer is associated with cardiovascular morbidity and mortality: A meta-analysis of population-based observational studies. PLoS One 2014;9:e107516. https://doi.org/10.1371/journal.pone.0107516

15. Bosco C, Bosnyak Z, Malmberg A, et al. Quantifying observational evidence for risk of fatal and nonfatal cardiovascular disease following androgen-deprivation therapy for prostate cancer: A meta-analysis. Eur Urol 2015;68:386-96. https://doi.org/10.1016/i.eururo.2014.11.039

16. Brodin E, Vikan T, Hansen JB, et al. Testosterone, hemostasis, and cardiovascular diseases in men. Semin Thromb Hemost 2011;37:87-94. https://doi.org/10.1055/s-0030-1270075

17. Malan NT, von Kanel R, Schutte AE, et al. Testosterone and acute stress are associated with fibrinogen and von Willebrand factor in African men: The SABPA study. Int I Cardiol 2013;168:4638-42. https://doi.org/10.1016/i.ijcard.2013.07.191

18. Timp JF, Braekkan SK, Versteeg HH, et al. Epidemiology of cancer-associated venous thrombosis. Blood 2013;122:1712-23. https://doi.org/10.1182/blood-2013-04-460121

19. Ehdaie $\mathrm{B}$, Atoria $\mathrm{CL}$, Gupta $\mathrm{A}$, et al. Androgen deprivation and thromboembolic events in men with prostate cancer. Cancer 2012;118:3397-406. https://doi.org/10.1002/cncr.26623

20. Hu JC, Williams SB, O'Malley AJ, et al. Androgen-deprivation therapy for non-metastatic prostate cancer is associated with an increased risk of peripheral arterial disease and venous thromboembolism. Eur Urol 2012;61:1119-28. hitps://doi.org/10.1016/i.eururo.2012.01.045

21. Van Hemelrijck M, Adolfsson J, Garmo $H$, et al. Risk of thromboembolic diseases in men with prostate cancer: Results from the population-based PCBaSe Sweden. Lancet Oncol 2010;11:450-8. hitps://doi.org/10.1016/S1470-2045(10)70038-3
22. Toukh M, Siemens DR, Black A, et al. Thromboelastography identifies hypercoagulablilty and predicts thromboembolic complications in patients with prostate cancer. Thromb Res 2014;133:88-95. https://doi.org/10.1016/i.thromres.2013.10.007

23. Keating NL, O'Malley AJ, Smith MR. Diabetes and cardiovascular disease during androgen-deprivation therapy for prostate cancer. J Clin Oncol 2006;24:4448-56. https://doi.org/10.1200/JC0.2006.06.2497

24. Braga-Basaria M, Dobs AS, Muller DC, et al. Metabolic syndrome in men with prostate cancer undergoing long-term androgen-deprivation therapy. J Clin Oncol 2006;24:3979-83. https://doi.org/10.1200/ JC0.2006.05.9741

25. Tivesten A, Pinthus JH, Clarke N, et al. Cardiovascular risk with androgen-deprivation therapy for prostate cancer: Potential mechanisms. Urol Oncol 2015;33:464-75. https://doi.org/10.1016/i. urolonc.2015.05.030

26. Emerging Risk Factors Collaboration. Kaptoge S, Di Angelantonio E, Pennells L, et al. C-reactive protein, fibrinogen, and cardiovascular disease prediction. N Engl J Med 2012;367:1310-20. https://doi.org/10.1056/NEJMoal 107477

27. Ziaran S, Goncalves FM, Breza J Sr. Patients with prostate cancer treated by ADT have significantly higher fibrinogenemia than healthy controls. World J Urol 2013;31:289-92. https://doi.org/10.1007/ s00345-012-0926-x

28. Chaturvedi $S$, Sidana $S$, Elson $P$, et al. Symptomatic and incidental venous thromboembolic disease are both associated with mortality in patients with prostate cancer. PLoS One 2014;9:e94048. htrps://doi.org/10.1371/journal.pone.0094048

29. Klil-Drori AJ, Yin H, Tagalakis $\mathrm{V}$, et al. Androgen-deprivation therapy for prostate cancer and the risk of venous thromboembolism. Eur Urol 2016;70:56-61. https://doi.org/10.1016/i.eururo.2015.06.022

30. Khorana AA, Carrier M, Garcia DA, et al. Guidance for the prevention and treatment of cancer-associated venous thromboembolism. J Thromb Thrombolysis 2016;41:81-91. https://doi.org/10.1007/s1 1239015-1313-4

Correspondence: Dr. Maha Othman, Queen's University, Kingston, 0N, Canada; othman@queensu.ca 